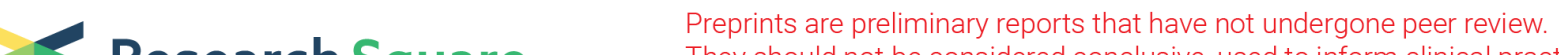 Research Square They should not be considered conclusive, used to inform clinical practice, or referenced by the media as validated information.
}

\section{A Sustainable Filtering Material for Efficient Removal of Volatile Organic Compounds From Their Aqueous Mixtures}

\section{Yong LV}

Yiwu Industrial and Commercial College

\section{Xiangju Xi}

Shaanxi University of Science and Technology

\section{Yijiao Xue}

Shaanxi University of Science and Technology

\section{Feng Jiang}

Zhejiang Shunpu New Material Technology Ltd.

\section{Xianqi Zhu}

Zhejiang Shunpu New Material Technology Ltd.

\section{Lei Dai ( $\nabla$ dailei_ju@hotmail.com )}

Shaanxi University of Science and Technology https://orcid.org/0000-0002-9011-2246

\section{Zhirong Chen}

Zhejiang University

\section{Research Article}

Keywords: cellulose nanofibers, cationic guar gum, self-assembling, hydrogel, volatile organic compounds (VOCs).

Posted Date: February 10th, 2021

DOI: https://doi.org/10.21203/rs.3.rs-162148/v1

License: (c) (1) This work is licensed under a Creative Commons Attribution 4.0 International License. Read Full License 


\section{Abstract}

Volatile organic compounds (VOCs) are hazardous to the environment and human health. Thus, tremendous effort has gone into developing effective/efficient techniques for VOCs separation from their aqueous mixtures. In this work, a simple and versatile strategy was proposed to fabricate a new material for hydrophobic volatile organic compounds (VOCs) separation, in which a self-assembling hydrogel consisting of TEMPO-oxidized cellulose nanofibers (TOCN) and cationic guar gum (CGG) was used. Specifically, TOCN and CGG were deposited onto a filter paper via a layer-by-layer procedure, resulting in a TOCN/CGG hydrogel-coated filtering material, in which no crosslinking agent was used. The hydrogel is spontaneously formed by electrostatic interactions and hydrogen bonding. The as-prepared filtering material exhibits oil-repellence and underwater oleophobicity resulting from the formation of hydration layer on hydrogel, thus demonstrating good VOCs separation performance. One-layer of TOCN/CGG hydrogel coating (dried mass of $0.032 \mathrm{~g} \cdot \mathrm{m}^{-2}$ ) was proved to realize the VOCs separation (separation efficiency of around $99 \%$ and water flux of as high as $905.14 \mathrm{~L} \cdot \mathrm{m}^{-2} \cdot \mathrm{h}^{-1}$ ). Further increase of the hydrogel layers has negligible influence on the separation efficiency although it decreases the water flux. This filtering material also exhibits good stability during recycling. Consequently, this TOCN/CGG selfassembling hydrogel-coated filtering material has great potential in VOCs-contaminated wastewater treatment.

\section{Introduction}

Organic solvents and its products are widely used in industrial activity, which results in large amounts of organic solvents-contaminated wastewater that seriously threatens the global environment and even causes human health issues (Rutkiewicz et al. 2010, Kang et al. 2019, Perego et al. 2013, Levchuk et al. 2014). Among organic solvents, volatile organic compounds (VOCs) are largely used when preparing various products, such as paints, adhesives and petroleum products (Fei et al. 2018, Sampranpiboon et al. 2000, Yoshida and Cohen 2004, Zhou et al. 2013, Kujawa et al. 2015). Nonetheless, VOCs are serious precursors of PM 2.5 and are also the main components of photochemical smog for they are very volatile at room temperature and pressure (An 2004, Farhadian et al. 2008, Hassan and Sorial 2010). The VOCs emission continuously rises with the rapid industrial development, and they constitute around $7 \%$ of the atmospheric pollutants (Delhoménie and Heitz 2005). Thus, it is urgent to eliminate or recover VOCs from wastewater.VOCs-contaminated wastewater treatment can be implemented with various techniques, such as adsorption, distillation, air stripping and membrane technology (Urkiaga et al. 2002, Zheng et al. 2013, Gallego et al. 2014). However, the separation efficiency of many existing techniques is still low, and some complex instruments also limit the practical application of these techniques (Zhu et al. 2015, 2016, Tummons et al. 2016). In this context, novel materials with wettability stand out as a promising alternative, owing to their good performance in separating oil/water two-phase mixtures. Hydrogels, which consist of three-dimensional polymer networks filled with abundant water inside their interstitial space, are a kind of ideal superwetting material for oily wastewater treatment (Dai et al. 2019a, b, Xu et al. 2014, Su et al. 2019, Sun et al. 2021). 
Hydrogels are generally prepared with crosslinked hydrophilic polymers. Cellulose (Rohrbach et al. 2014), guar gum (Dai et al. 2017) and chitosan (Tu et al. 2017) have been widely used to fabricate hydrogels due to their abundance, renewability, and functionality, which perfectly meets the requirements of "green" and sustainable development. Moreover, hydrogels without chemical crosslinkers are preferred in many situations to avoid the toxicity from the crosslinkers. In this sense, self-assembling hydrogels without the use of extraneous crosslinkers have attracted a lot of attention. We previously reported self-assembling hydrogels made of guar gum and nanocellulose (Dai et al. 2019a, b).

In this work, we proposed to use TEMPO-oxidized cellulose nanofibers (TOCN) and cationic guar gum (CGG) to coat filter papers through a layer-by-layer procedure. Hydrogel-coated filtering material can be obtained since TOCN and CGG autonomously generate a hydrogel due to the electrostatic interactions and hydrogen bonding between them, without the need of any extraneous crosslinkers. The layer-by-layer process also allows the modulation of the filtering material. The performance of TOCN/CGG selfassembling hydrogel-coated filtering material in removing VOCs from their aqueous mixtures was thoroughly studied.

\section{Experimental \\ 2.1 Materials}

Chemicals including N-heptane, petroleum ether, styrene, etc., were bought from Aladdin (Shanghai, China). TOCN was purchased from Tianjin Woodelf Biotechnology Co., Ltd. (China), and cationic guar gum was sourced from a local company. Filter papers (pore size of 80-120 $\mu \mathrm{m}$ ) were obtained from Hangzhou Newstar Paper (China).

\subsection{Fabrication of TOCN/CGG hydrogel-coated filtering material}

The filtering material was fabricated via the assembling of TOCN/CGG hydrogel onto filter papers as a coating. In detail, TOCN and CGG with both concentrations of $0.01 \mathrm{wt} \%$ were sequentially deposited onto the filter paper via a layer-by-layer process. One layer of hydrogel coating (dried mass of $0.032 \mathrm{~g} \cdot \mathrm{m}^{-2}$ ) consisted of one layer of TOCN and one layer of CGG. The filtering materials of different layers of hydrogel coating were obtained by repeating the deposition procedure, and all these filtering materials were air-dried at room temperature.

\subsection{Volatile organic compounds (VOCs) separation}

$\mathrm{N}$-heptane, petroleum ether, styrene, etc. were used to implement the VOCs separation. VOCs aqueous mixtures were prepared by mixing one type of VOCs with equivalent deionized water. A piece of TOCN/CGG hydrogel-coated filtering material was mounted onto a glass filter to separate the abovementioned VOCs aqueous mixtures, during which the gravity drove the separation process. The separation efficiency $(E)$ was calculated as the weight ratio of collected water and initial water in the 
mixture. Water flux was calculated with the volume of collected water in a time interval. Filtering materials with different hydrogel layers (1 to 4 layers) were used for the separation study. Moreover, different VOCs, including petroleum ether, styrene, n-hexane, ethylbenzene, and turpentine, were adopted to demonstrate the separation performance of this TOCN/CGG hydrogel-coated filtering material for VOCs.

\subsection{Characterizations}

The quantitative determination of total organic carbon (TOC) in the filtrate was performed with a total organic carbon (TOC) analyser (Vario TOC). The samples were collected and sealed and were sent for analysis within 24 hours to prevent evaporation. The morphology and structure of the as-prepared filtering material were observed with a scanning electron microscope (SEM, JEOL 6400) with the acceleration voltage of $15 \mathrm{kV}$, before which the specimens were dried at ambient conditions and sputtercoated with gold.

\section{Results And Discussion}

TOCN and CGG were coated on the filter paper through a layer-by-layer deposition process. Thanks to the non-covalent interactions (electrostatic interactions and H-bonding) between TOCN and CGG, a selfassembled hydrogel can be easily and instantly formed on the filter paper without any cross-linking agent. Furthermore, no adhesive agents are required to attach the hydrogel onto the filter paper, for both components inherently have good affinity with cellulosic paper. The TOCN/CGG hydrogel-coated filtering material is shown in Fig. 1a. This proposed technique shows advantages over some reported counterparts. For instance, the adhesive agent was needed to bind the precursor with the substrate (i.e. steel mesh) in the preparation of polyacrylamide (PAM) hydrogel-coated steel mesh, and some time (90 to $120 \mathrm{~min}$ ) was needed for the hydrogel formation (Xue et al. 2011). The oil repellence performance of the hydrogel-coated filtering material was characterized with silicone oil and the results are shown in Fig. 1b. The silicone oil droplet nimbly flows across the filtering material indicating the low adhesion force between oil droplet and filtering material. Thus, the TOCN/CGG hydrogel-coated filtering material has good oil repellence property. The good underwater oleophobic performance was also studied with silicone oil (Fig. 1C). The oil repellence and oleophobic properties of the resultant TOCN/CGG hydrogelcoated filtering material prove its potential in separating VOCs from their aqueous mixtures.

The TOCN/CGG hydrogel-coated filtering material was analyzed with different VOCs to show its separation performance. The setup is illustrated in Fig. 2. As shown, the TOCN/CGG hydrogel-coated filtering material was mounted in the glass filter into which the petroleum ether/water mixture was poured to start the separation under the gravity force. Obviously, the water passed through the filtering material while the petroleum ether was prevented from passing through. Thus, the VOCs separation from aqueous solution was realized.

Total organic carbon (TOC), representing the total organic substances in water, is an important indicator for evaluating the degree of pollution of water bodies by organic substances. The filtered water was 
analyzed in terms of total organic carbon (TOC) content to further confirm the blockage of petroleum ether from passing through the hydrogel-coated filtering material. As listed in Table 1, the TOC content is as low as $1.16 \mathrm{mg} \cdot \mathrm{L}^{-1}$ when one-layer hydrogel-coated filtering material is used, which indicates that hydrogel modification is very effective in blocking VOCs. The one-layer hydrogel-coated filtering material exhibits a separation efficiency of ca. $99.06 \%$. And the separation efficiency negligibly changes with the increase of the hydrogel layer. Nevertheless, the water flux decreases from 863.77 to $306.85 \mathrm{~L} \cdot \mathrm{m}^{-2} \cdot \mathrm{h}^{-1}$ as the hydrogel layer increases from 1 to 4 , which might be caused by the increased hydrogel thickness, slowing down the water penetrating speed.

Total organic carbon (TOC), representing the total organic substances in water, is an important indicator for evaluating the degree of pollution of water bodies by organic substances. The filtered water was analyzed in terms of total organic carbon (TOC) content to further confirm the blockage of petroleum ether from passing through the hydrogel-coated filtering material. In particular, the water sample was taken out of the water phase in the oil/water mixture as the control. As listed in Table 1, the TOC content is as low as $1.16 \mathrm{mg} \cdot \mathrm{L}^{-1}$ when one-layer hydrogel-coated filtering material is used, which indicates that hydrogel modification is very effective in blocking VOCs. The one-layer hydrogel-coated filtering material exhibits a separation efficiency of ca. $99.06 \%$. And the separation efficiency negligibly changes with the increase of the hydrogel layer. Nevertheless, the water flux decreases from 863.77 to $306.85 \mathrm{~L} \cdot \mathrm{m}^{-2} \cdot \mathrm{h}^{-1}$ as the hydrogel layer increases from 1 to 4 , which might be caused by the increased hydrogel thickness, slowing down the water penetrating speed.

Table 1

The data of TOCN/CGG hydrogel-coated filtering material in separating petroleum ether/water mixture.

\begin{tabular}{|llll|}
\hline Hydrogel Layer & $\begin{array}{l}\text { Total organic carbon } \\
\left(\mathbf{m g} \cdot \mathrm{L}^{-1}\right) *\end{array}$ & $\begin{array}{l}\text { Separation efficiency } \\
(\%)^{*}\end{array}$ & $\begin{array}{l}\text { Water flux } \\
\left(\mathbf{L} \cdot \mathbf{m}^{-\mathbf{2}} \cdot \mathrm{h}^{-1} \mathbf{)}\right.\end{array}$ \\
\hline Control & 1.60 & $/$ & $/$ \\
\hline 1 & 1.16 & 99.06 & 863.77 \\
\hline 3 & 1.00 & 99.35 & 616.48 \\
\hline 4 & 0.95 & 99.17 & 328.91 \\
\hline *Average data is reported. & 99.10 & 306.85 \\
\hline
\end{tabular}

Based on the aforementioned separation efficiency results, 1-layer hydrogel modification is preferred for the filtering material design. However, the thinner the hydrogel layer, the easier of the filtering material to be worn-out. In this context, the recyclability of one-layer hydrogel-coated filtering material was studied, in which petroleum ether was used as a model VOCs (Fig. 3). The results show that the separation efficiency maintains around $99 \%$ during the recycles. 
To further determine the VOCs separation performance of TOCN/CGG hydrogel-coated filtering material, six typical VOCs (i.e. n-heptane, petroleum ether, styrene, n-hexane, ethylbenzene, and turpentine) were used to perform the separation analysis. Figure 4a indicates that the one-layer hydrogel-coated filtering material shows the high separation efficiency for different VOCs (98.32\% (n-heptane), $99.06 \%$ (petroleum ether), $99.02 \%$ (styrene), 98.88\% (n-hexane), 98.76\% (ethylbenzene) and 98.72\% (turpentine oil)). Furthermore, the as-prepared hydrogel-coated filtering materials with different numbers of hydrogel layer were studied in this work, and the results are shown in Fig. 4b-4d. These filtering materials all show high oil/water separation efficiency and their separation efficiency is not significantly affected by the numbers of hydrogel layer, indicating that the TOCN/CGG hydrogel-coated filtering materials possess oleophobic properties once an integrated hydrogel layer is generated.

The effect of the number of hydrogel layer on water flux in the VOCs/water separation was also analyzed. Figure 5 demonstrates that the layer number of hydrogel coating significantly affects the water flux in the separation process. For instance, the water flux significantly decreases as the layer number of hydrogel coating increases in separating $n$-heptane/water mixtures (Fig. 5a). It decreases from 848.53 to 271.27 $\mathrm{L} \cdot \mathrm{m}^{-2} \cdot \mathrm{h}^{-1}$ as the hydrogel coating increases from 1 to 4 layers. The same trend appears in separating other VOCs/water mixtures (Fig. 5b-f). The decreased water reflux can be attributed to the decreased pores and voids on the surface of filtering material, as well as the increased tortuosity inside the hydrogel as the number of hydrogel layer increases.

To further clarify this, SEM was used to observe the morphology and structure of TOCN/CGG hydrogelcoated filtering material (Fig. 6). As shown, the original filter paper exhibits typical porous structures (Fig. 6a) while the hydrogel coating covers a lot of pores and voids, thus remarkably decreasing the size of water passage (Fig. 6b). This change is in accordance with the nanofibrillated cellulose hydrogelcoated filter papers reported by Rohrbach et al. (Rohrbach et al. 2014). It is easy to imagine that the hydrogel thickness increases as the number of hydrogel layer increases, which decreases the water flux because the water penetration speed is slowed down as a result of the decreased pores and voids, as well as the increased tortuosity.

\section{Conclusions}

In this study, a sustainable TOCN/CGG hydrogel-coated filtering material is demonstrated to efficiently separate VOCs from their water mixtures. The as-prepared one-layer hydrogel-coated filtering material showed high VOCs/water separation efficiency of around $99 \%$, with the hydrogel coating amount of only $0.032 \mathrm{~g} \cdot \mathrm{m}^{-2}$ (dried mass). Meanwhile, the maximum water flux during the separation reaches $905.14 \mathrm{~L} \cdot \mathrm{m}^{-}$

$2 \cdot h^{-1}$, driven by the gravity force. The increase of the number of hydrogel layer decreases the water flux for the water penetration speed is slowed down as the hydrogel thickness increases. This filtering material also exhibits good stability for it maintains the separation efficiency of around $99.0 \%$ after 20 times of recycling. The outstanding performance demonstrates the potential of this filtering material in treating VOCs-containing wastewater in the future. 


\section{Declarations}

\section{Acknowledgments}

The authors acknowledge the financial support from the National Natural Science Foundation of China (grant number 31901265), China Postdoctoral Science Foundation (grant number 2019M662061), Natural Science Foundation of Shaanxi Province (grant number 2019JQ-784) and Postdoctoral Science Foundation of Zhejiang Province (grant number zj2019135).

\section{Conflicts of interest}

There is no conflict of interest.

\section{References}

1. An YJ (2004). Toxicity of benzene, toluene, ethylbenzene, and xylene (BTEX) mixtures to Sorghum bicolor and Cucumis sativus. Bulletin of Environmental Contamination and Toxicology, 72(5), 10061011.

2. Dai L, Cheng T, Wang Y, Wanpoog B, Duan C, Ke H, Ni Y (2019a). A self-assembling guar gum hydrogel for efficient oil/water separation in harsh environments. Separation and Purification Technology, 225, 129-135.

3. Dai L, Cheng T, Wang Y, Lu H, Nie S, He H, Ni Y (2019b). Injectable all-polysaccharide self-assembling hydrogel: a promising scaffold for localized therapeutic proteins. Cellulose, 26(11), 6891-6901.

4. Dai L, Wang B, An X, Zhang L, Khan A, Ni Y (2017). Oil/water interfaces of guar gum-based biopolymer hydrogels and application to their separation. Carbohydrate polymers, 169, 9-15.

5. Delhoménie MC, Heitz M (2005). Biofiltration of air: a review. Critical Reviews in Biotechnology, 25(12), 53-72.

6. Farhadian M, Duchez D, Vachelard C, Larroche C (2008). Monoaromatics removal from polluted water through bioreactors-A review. Water Research, 42(6-7), 1325-1341.

7. Fan F, Levente C, Gyorgy S, Christopher F. B (2018). Robust covalently cross-linked polybenzimidazole/graphene oxide membranes for high-flux organic solvent nanofiltration. ACS Applied Materials \& Interfaces,10(18), 16140-16147.

8. Gallego E, Perales JF, Roca FJ, Guardino X (2014). Surface emission determination of volatile organic compounds (VOC) from a closed industrial waste landfill using a self-designed static flux chamber. Science of the Total Environment, 470, 587-599.

9. Hassan AA, Sorial GA (2010). Removal of benzene under acidic conditions in a controlled trickle bed air biofilter. Journal of Hazardous Materials, 184(1-3), 345-349.

10. Kang L, Li J, Zeng J, Gao W, Xu J, Cheng Z, Chen K and Wang B (2019). A water solvent-assisted condensation polymerization strategy of superhydrophobic lignocellulosic fibers for efficient oil/water separation. Journal of Materials Chemistry A, 7(27), 16447-16457. 
11. Kujawa J, Cerneaux S, Kujawski W (2015). Removal of hazardous volatile organic compounds from water by vacuum pervaporation with hydrophobic ceramic membranes. Journal of Membrane Science, 474, 11-19.

12. Levchuk I, Bhatnagar A, Sillanpaa M (2014). Overview of technologies for removal of methyl tertbutyl ether (MTBE) from water. Science of the Total Environment, 476, 415-433.

13. Xu L, Liu N, Cao Y, Lu F, Chen Y, Zhang X, Wei Y (2014). Mercury ion responsive wettability and oil/water separation. ACS Applied Materials \& Interfaces, 6(16), 13324-13329.

14. Perego C, Bagatin R, Tagliabue M, Vignola R (2013). Zeolites and related mesoporous materials for multi-talented environmental solutions. Microporous and Mesoporous Materials, 166, 37-49.

15. Rohrbach K, Li Y, Zhu H, Liu Z, Dai J, Andreasen J, Hu L (2014). A cellulose based hydrophilic oleophobic hydrated filter for water/oil separation. Chemical Communications, 50(87), 13296-13299.

16. Rutkiewicz I, Kujawski W, Namieśnik J (2010). Pervaporation of volatile organohalogen compounds through polydimethylsiloxane membrane. Desalination, 264(1-2), 160-164.

17. Sampranpiboon P, Jiraratananon R, Uttapap D, Feng X, Huang RYM (2000). Pervaporation separation of ethyl butyrate and isopropanol with polyether block amide (PEBA) membranes. Journal of Membrane Science, 173(1), 53-59.

18. Su M, Liu Y, Li S, Fang Z, He B, Zhang Y, He P (2019). A rubber-like, underwater superoleophobic hydrogel for efficient oil/water separation. Chemical Engineering Journal, 361, 364-372.

19. Sun Y, Chu Y, Wu W, Xiao H (2021). Nanocellulose-based lightweight porous materials: A review. Carbohydrate Polymers, 255, 117489.

20. Tu H, Yu Y, Chen J, Shi X, Zhou J, Deng H, Du Y (2017). Highly cost-effective and high-strength hydrogels as dye adsorbents from natural polymers: chitosan and cellulose. Polymer Chemistry, 8(19), 2913-2921.

21. Tummons EN, Tarabara VV, Chew JW, Fane AG (2016). Behavior of oil droplets at the membrane surface during crossflow microfiltration of oil-water emulsions. Journal of Membrane Science, 500, 211-224.

22. Urkiaga A, Bolano N, De Las Fuentes, L (2002). Removal of micropollutants in aqueous streams by organophilic pervaporation. Desalination, 149(1-3), 55-60.

23. Xue Z, Wang S, Lin L, Chen L, Liu M, Feng L, Jiang L (2011). A novel superhydrophilic and underwater superoleophobic hydrogel-coated mesh for oil/water separation. Advanced Materials, 23(37), 42704273.

24. Yoshida W, Cohen Y (2004). Removal of methyl tert-butyl ether from water by pervaporation using ceramic-supported polymer membranes. Journal of Membrane Science, 229(1-2), 27-32.

25. Zheng S, Shen C, Alunbate M, Deng J, Wang L, Han Z, Tang, H (2013). Discovery of VOC-compliant TEOS sol and its application to $\mathrm{SiO}_{2}$ /novolac hybrid coatings. Progress in Organic Coatings, 76(2-3), 425-431. 
26. Zhou K, Zhang QG, Han GL, Zhu AM, Liu QL (2013). Pervaporation of water-ethanol and methanolMTBE mixtures using poly (vinyl alcohol)/cellulose acetate blended membranes. Journal of Membrane Science, 448, 93-101.

27. Zhu L, Chen M, Dong Y, Tang CY, Huang A, Li L (2016). A low-cost mullite-titania composite ceramic hollow fiber microfiltration membrane for highly efficient separation of oil-in-water emulsion. Water Research, 90, 277-285.

28. Zhu Y, Xie W, Li J, Xing T, Jin J (2015). pH-Induced non-fouling membrane for effective separation of oil-in-water emulsion. Journal of Membrane Science, 477, 131-138.

\section{Figures}
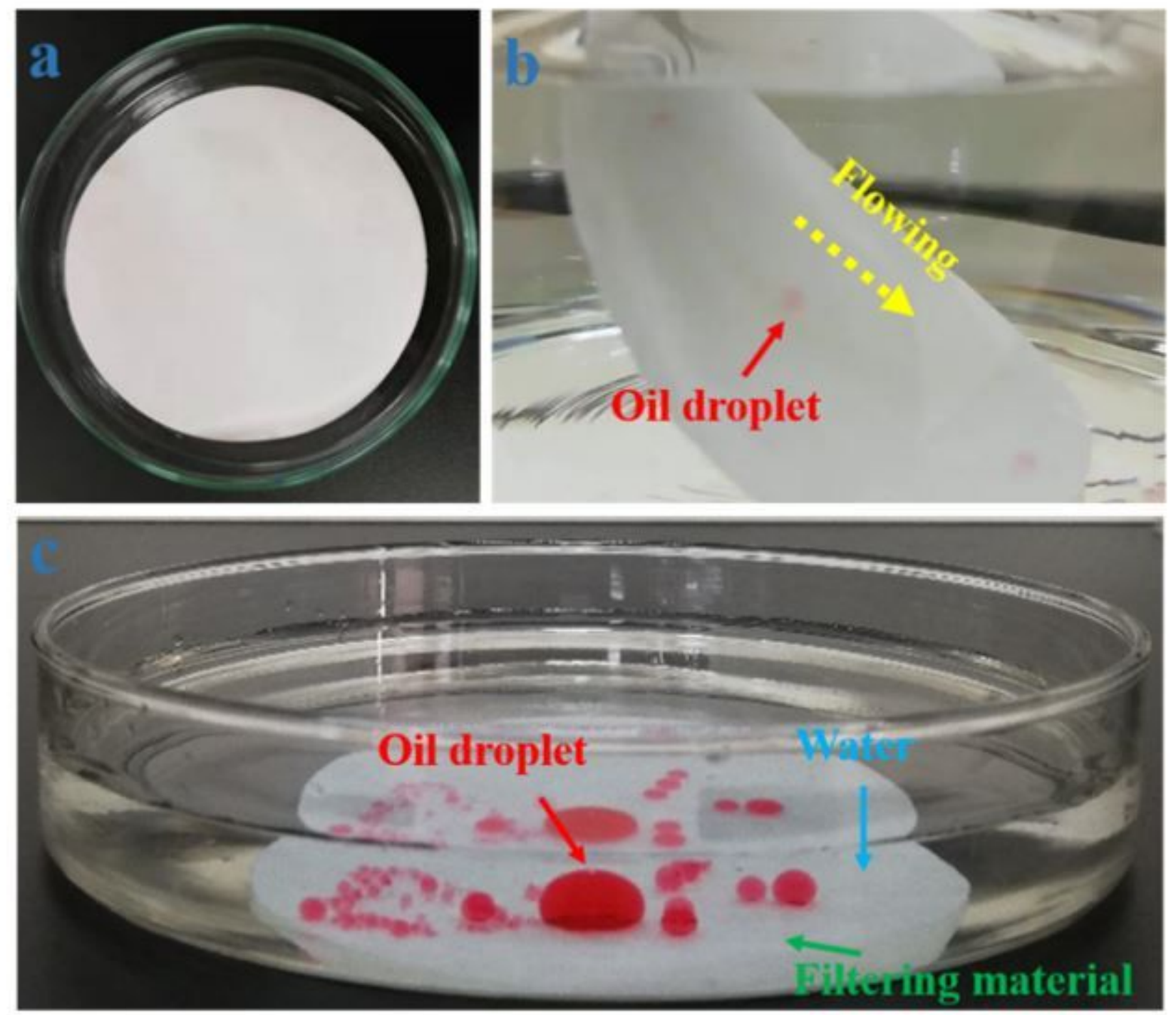

\section{Figure 1}

TOCN/CGG hydrogel-coated filtering material: its digital photo (a), oil repellence (b) and underwater oleophobicity (c). 

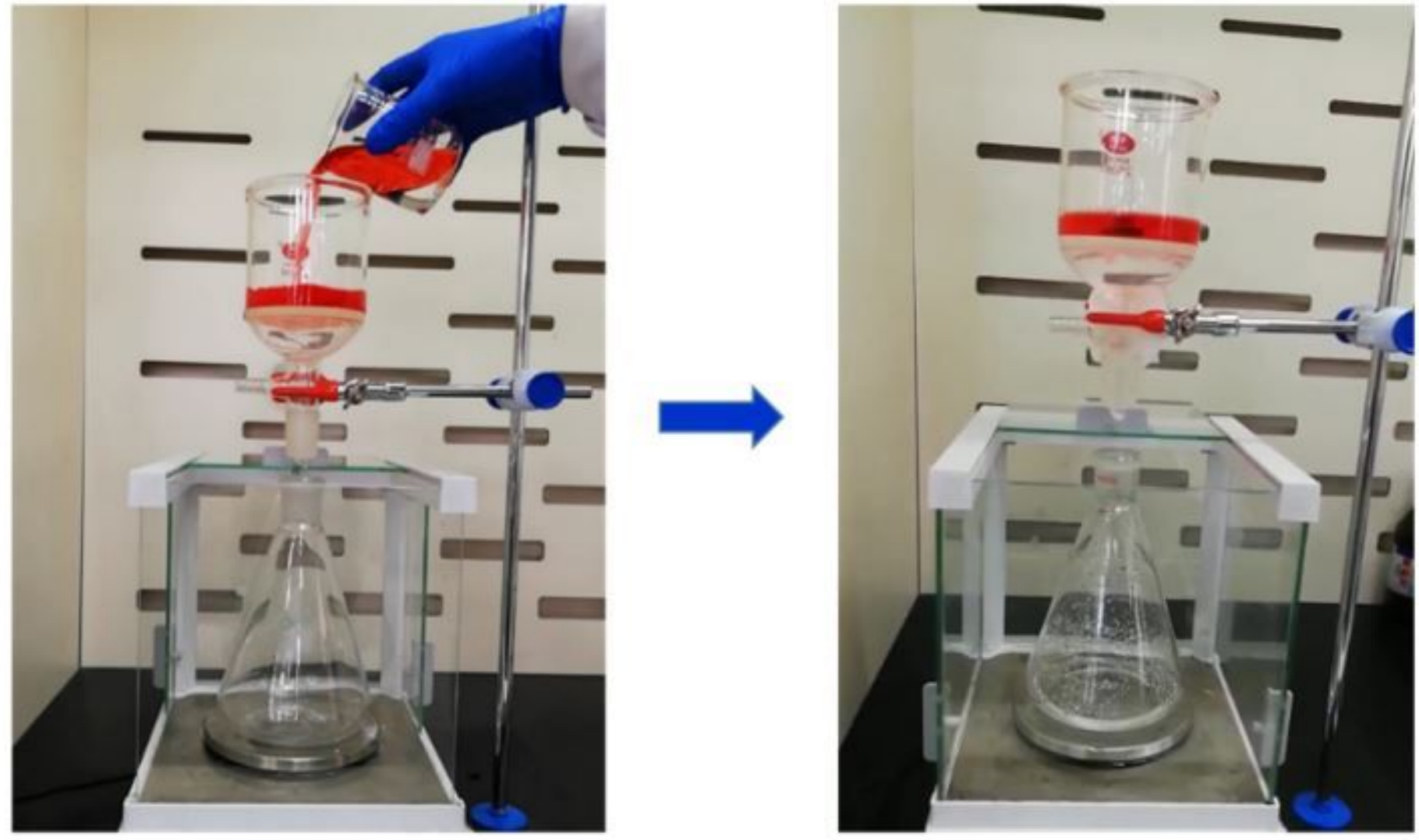

Figure 2

The setup for VOCs separation from aqueous solutions (oil red O dyed petroleum ether used as a VOCs model). 


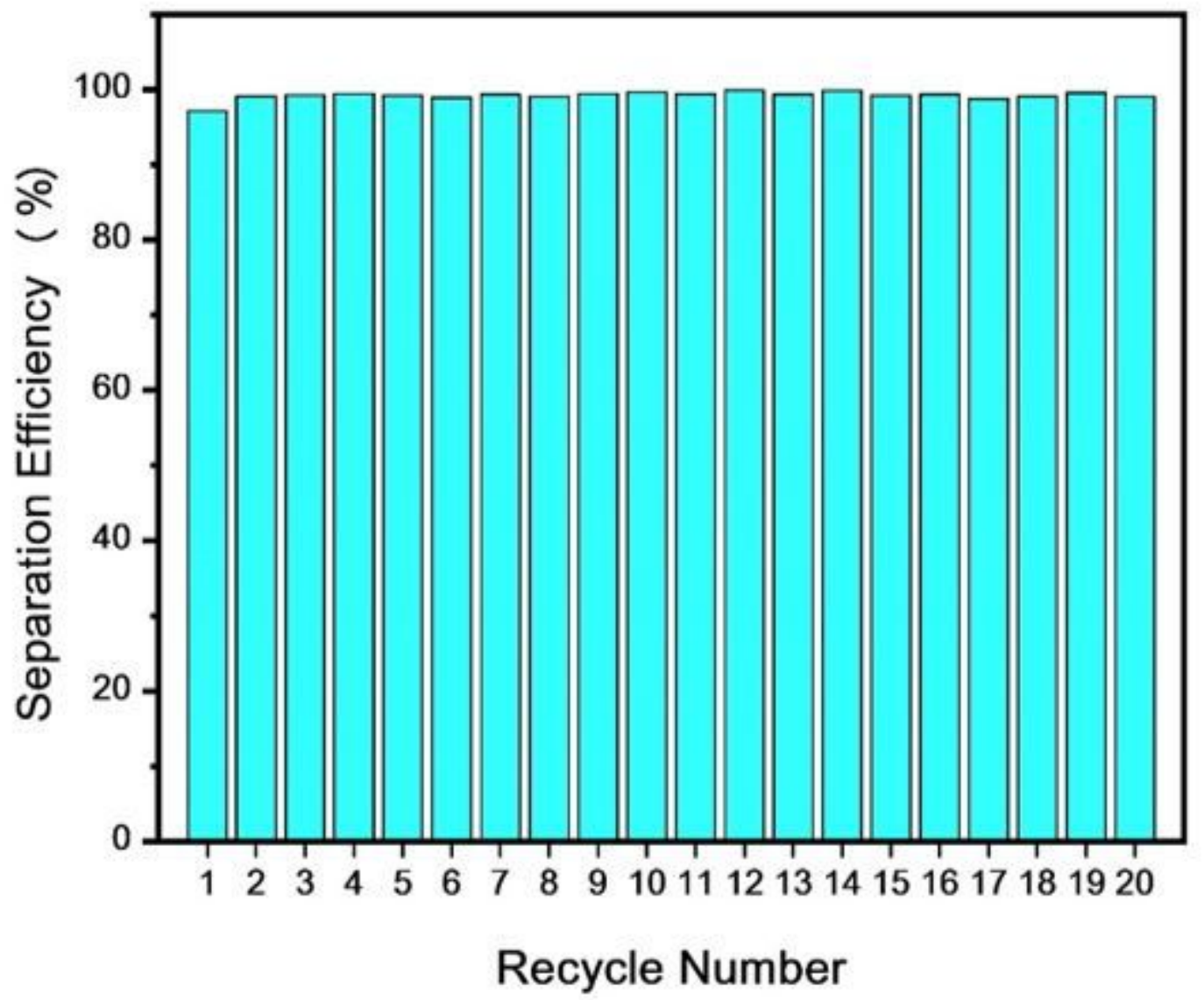

Figure 3

Recyclability of the TOCN/CGG hydrogel-coated filtering material in separating petroleum ether/water mixture. 

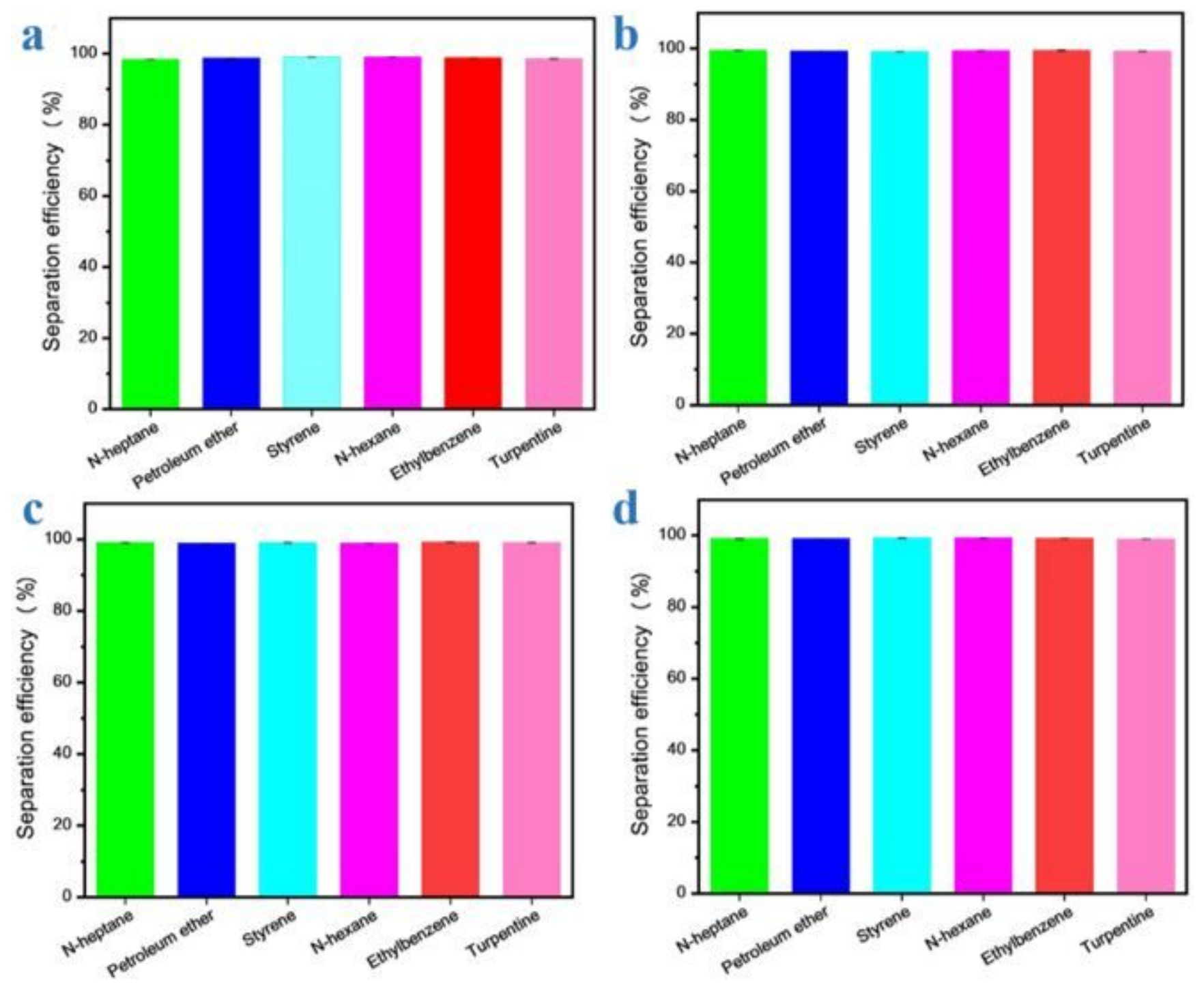

\section{Figure 4}

Separation efficiency of the TOCN/CGG hydrogel-coated filtering material with different layers of hydrogel (one-layer (a); two-layer (b); three-layer (c); four-layer (d)). 

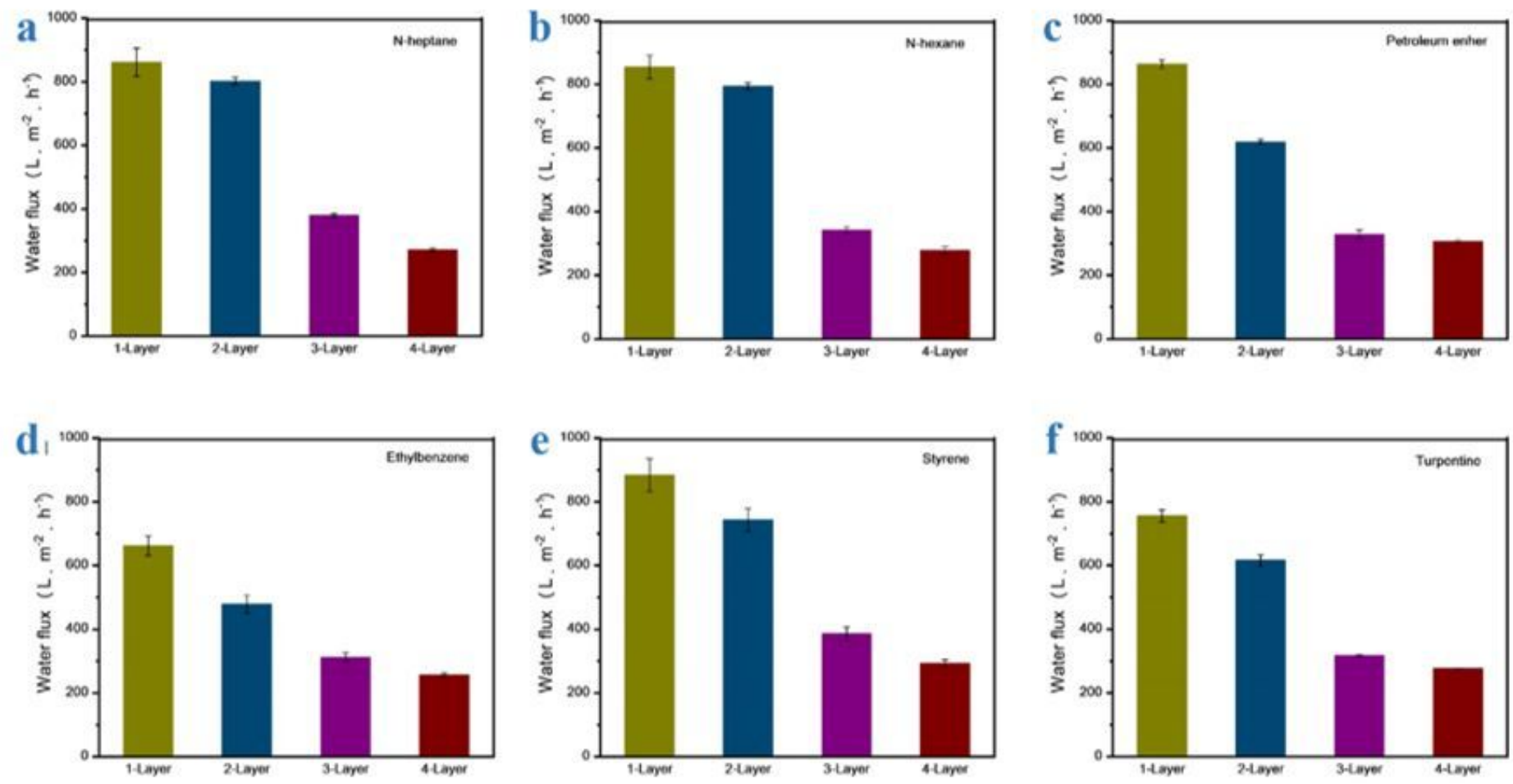

\section{Figure 5}

Water flux of the TOCN/CGG hydrogel-coated filtering material in separating VOCs/water mixtures (nheptane (a); n-hexane (b); petroleum ether oil (c); ethylbenzene (d); styrene (e); turpentine (f)).
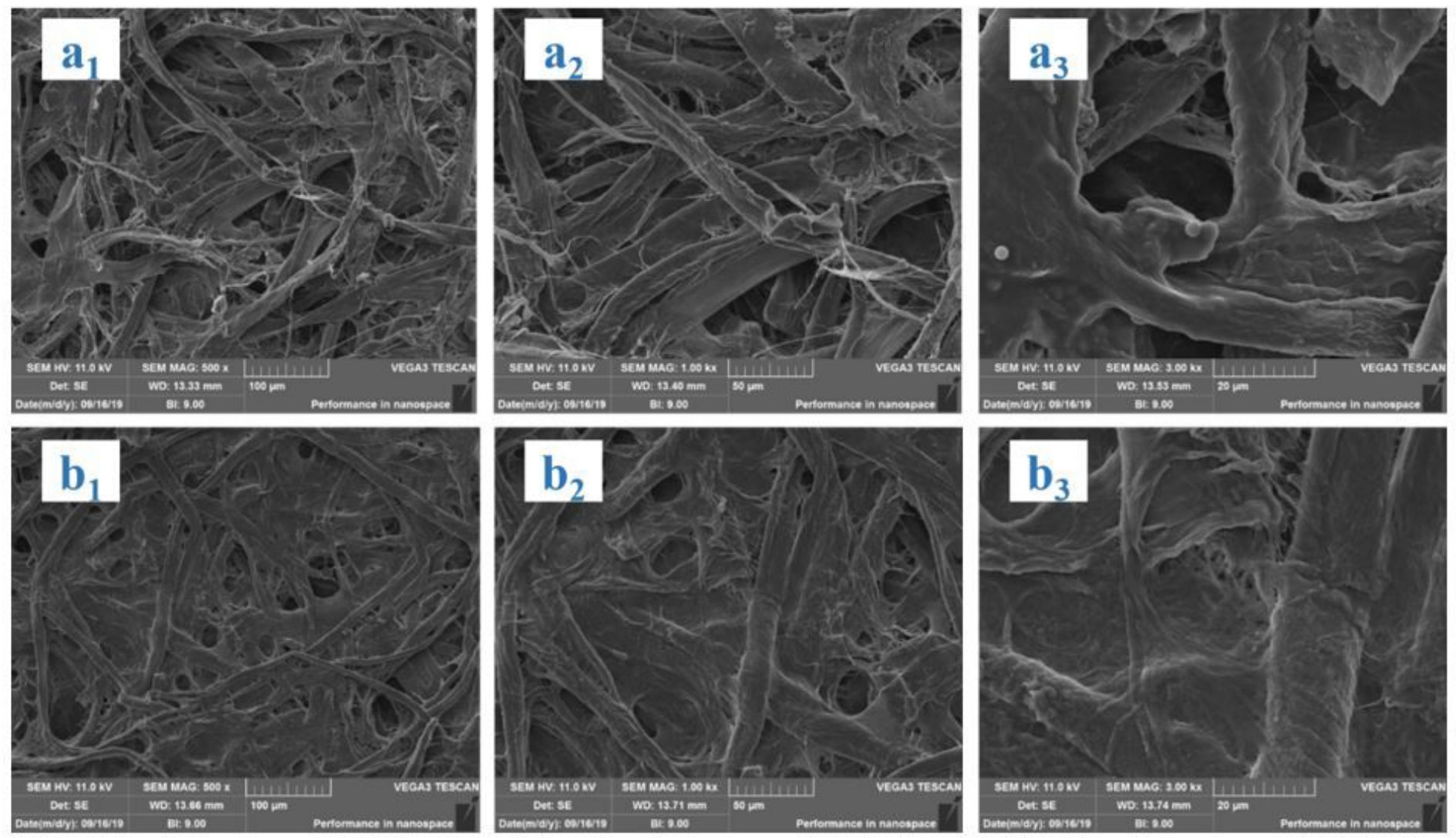
Figure 6

SEM images of original (a) and four-layer TOCN/CGG hydrogel-coated filtering material (b). 